\title{
Population genetic structure of the major malaria vector Anopheles funestus S.S. and allied species in southern Africa
}

Kwang Shik Choi ${ }^{1,2^{*}}$, Lizette L Koekemoer ${ }^{1,2}$ and Maureen Coetzee ${ }^{1,2}$

\begin{abstract}
Background: Anopheles funestus s.S., one of the major malaria vectors in sub-Saharan Africa, belongs to a group of eleven African species that are morphologically similar at the adult stage, most of which do not transmit malaria. The population structure of An. funestus based on mitochondrial DNA data led to the description of two cryptic subdivisions, clade I widespread throughout Africa and clade II known only from Mozambique and Madagascar. In this study, we investigated five common members of the Anopheles funestus group in southern Africa in order to determine relationships within and between species.

Methods: A total of 155 specimens of An. funestus, An. parensis, An. vaneedeni, An. funestus-like and An. rivulorum from South Africa, Mozambique and Malawi were used for the study. The population genetic structure was assessed within and between populations using mitochondrial DNA.

Results: The phylogenetic trees revealed three main lineages: 1) An. rivulorum; 2) An. funestus-like clade I and An. parensis clade II; and 3) An. funestus clades I and II, An. funestus-like clade II, An. parensis clade I and An. vaneedeni clades I and II. Within An. funestus, 32 specimens from Mozambique consisted of $40.6 \%$ clade I and $59.4 \%$ clade II while all 21 individuals from Malawi were clade I. In the analysis of mitochondrial DNA sequences, there were 37 polymorphic sites and 9 fixed different nucleotides for ND5 and 21 polymorphic sites and 6 fixed different nucleotides for $\mathrm{CO}$ l between the two An. funestus clades. The results for COl supported the ND5 analysis.

Conclusion: This is the first report comparing An. funestus group species including An. funestus clades I and II and the new species An. funestus-like. Anopheles funestus clade I is separated from the rest of the members of the An. funestus subgroup and An. funestus-like is distinctly distributed from the other species in this study. However, there were two clades for An. funestus-like, An. parensis and An. vaneedeni. Further investigations are needed to determine what these results mean in terms of the specific status of the clades within each taxon and whether this has any epidemiological implications for malaria transmission.
\end{abstract}

Keywords: Anopheles funestus, Clade, ND5, COl, Phylogeny

\section{Background}

Malaria due to Plasmodium falciparum is a major cause of morbidity and mortality in children and pregnant women. The World Health Organization estimates that there were 216 million malaria cases in 2010, with 655,000 deaths [1]. Malaria also poses a risk to travelers

\footnotetext{
* Correspondence: kwangshik@gmail.com

'Malaria Entomology Research Unit, School of Pathology, Faculty of Health Sciences, University of the Witwatersrand, Johannesburg, South Africa ${ }^{2}$ Vector Control Reference Laboratory, Centre for Opportunistic, Tropical and Hospital Infections, National Institute for Communicable Diseases, National Health Laboratory Service, Johannesburg, South Africa
}

and immigrants, with imported cases increasing in nonendemic areas [2]. The treatment and control of malaria has become more difficult with the spread of drugresistant strains of parasites [3] and insecticide-resistant mosquito vectors [4-6].

Depending on the vectorial capacity and competence of local mosquitoes, transmission intensity of human malaria varies across Africa. Only a limited number of Anopheles species are able to transmit Plasmodium malaria to humans [7] and Anopheles funestus Giles is one of the three major malaria vectors in Africa. It is the

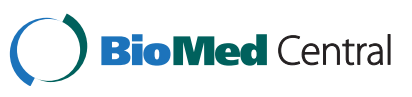


nominal member of a large group of mosquitoes that consists of at least eleven African species that are morphologically similar at the adult stage [8,9]: An. funestus, An. funestus-like, An. vaneedeni, An. parensis, An. aruni, An. confusus, An. brucei, An. fuscivenosus, An. rivulorum, An. rivulorum-like and An. leesoni [7-11]. Anopheles funestus is the most anthropophilic and endophilic member of the group [7], while the others are mainly zoophilic and not involved in malaria transmission except for $A n$. rivulorum, which is a minor vector in Tanzania [12] and An. vaneedeni which is a possible vector under laboratory conditions [13].

Anopheles funestus and An. rivulorum are widely distributed throughout sub-Saharan Africa [7,9]. The extent of the distribution of the other members in the group is largely unknown or they are more localized. Anopheles funestus-like has so far only been recorded from Malawi [11] and An. rivulorum-like occurs in Cameroon and Burkina Faso but might extend to western and central Africa [9,14]. Anopheles parensis is found in eastern Africa, Swaziland and South Africa. Anopheles confusus is distributed in eastern and southern Africa and An. vaneedeni is found in South Africa. Anopheles aruni, An. brucei and $A n$. fuscivenosus are extremely rare and found only in Zanzibar, Nigeria and Zimbabwe respectively [7,9].

A recent study of $A n$. funestus population structure based on NADH Dehydrogenase subunit 5 (ND5) mitochondrial DNA data [15] led to the description of two cryptic subdivisions, clade I which was found in all 11 African countries sampled and clade II found only in Mozambique and Madagascar. These two clades were differentiated by two fixed differences and an average of $2 \%$ divergence, which was thought to indicate that they have evolved independently for $\sim 1$ million years. Michel et al. $[15,16]$ also reported the existence of at least one main division between populations of $A n$. funestus on the basis of microsatellite allele frequencies. Furthermore, digestion of the internal transcribed spacer region 2 (ITS2) in the rDNA using restriction enzymes showed several different "types" within An. funestus [17,18] (see [19] for a review of the molecular systematics of An. funestus).
Understanding the characteristics of the species in the An. funestus group is necessary for effective malaria vector control programmes. The group may well be as complex and problematic as the An. gambiae complex $[19,20]$ given the results that we have from only the few population genetic studies that have been done to date. The aim of the present study was to expand previous work by examining inter- and intra-specific relationships between the five most common members of the $A n$. funestus group from southern Africa (An. funestus, An. funestus-like, An. parensis, An. rivulorum and An. vaneedeni), using mitochondrial markers and phylogenetic analysis.

\section{Methods}

\section{Mosquito samples}

Sampling information for the five species used in this study is given in Table 1. A total of 53 An. funestus specimens were collected resting inside houses from Mozambique $(n=32)$ in 2004 and Malawi $(n=21)$ in 2001. The collection of $26 \mathrm{An}$. parensis, $30 \mathrm{An}$. vaneedeni and 19 An. rivulorum specimens from South Africa were from outdoor $\mathrm{CO}_{2}$-baited traps between 2002 and 2008 . The 27 An. funestus-like specimens from Malawi were collected resting inside houses in 2007 and $F_{1}$ progenies from the specimens were used for this study. Different collection methods were used depending on the biology of each species to try and maximize species diversity.

\section{Laboratory methods}

All specimens were identified to species by standard rDNA PCR methods [11,21,22]. DNA samples were extracted from either single mosquitoes or available parts of mosquitoes using standard extraction protocols [23].

The modified primers for ND5 were from Michel et al. [16] and the primers for COI from Simon et al. [24]. The sequenced region for ND5 was confirmed with the data from Michel et al. [16]. The region was amplified using primers New ND5F (5'-AGA AAT CAA TAT ATA GAA GAA GAT T-3') and New ND5R (5'-TTC GAA TAT CTT GAG AAT TTT T-3') for ND5, and C1-

Table 1 Species, localities and the total numbers of specimens for each species

\begin{tabular}{|c|c|c|c|c|c|c|}
\hline & \multirow[t]{2}{*}{ Locality } & \multicolumn{5}{|c|}{ Species } \\
\hline & & An. funestus & An. funestus-like & An. parensis & An. rivulorum & An. vaneedeni \\
\hline Mozambique & Chibuto $\left(24^{\circ} 40^{\prime} \mathrm{S}, 33^{\circ} 33^{\prime} \mathrm{E}\right)$ & 32 & & & & \\
\hline \multirow[t]{2}{*}{ Malawi } & Karonga $\left(10^{\circ} 19^{\prime} \mathrm{S}, 34^{\circ} 08^{\prime} \mathrm{E}\right)$ & 11 & 27 & & & \\
\hline & Nkhota kota $\left(12^{\circ} 55^{\prime} \mathrm{S}, 34^{\circ} 18^{\prime} \mathrm{E}\right)$ & 10 & & & & \\
\hline \multirow[t]{4}{*}{ South Africa } & Mamfene $\left(27^{\circ} 23^{\prime} \mathrm{S}, 32^{\circ} 12^{\prime} \mathrm{E}\right)$ & & & 8 & & 12 \\
\hline & Ndumu $\left(27^{\circ} 02^{\prime} \mathrm{S}, 32^{\circ} 19^{\prime} \mathrm{E}\right)$ & & & 18 & & \\
\hline & Komatipoort $\left(25^{\circ} 26^{\prime} \mathrm{S}, 31^{\circ} 57^{\prime} \mathrm{E}\right)$ & & & & 19 & 12 \\
\hline & Giyani area $\left(23^{\circ} 15^{\prime} \mathrm{S}, 30^{\circ} 47^{\prime} \mathrm{E}\right)$ & & & & & 6 \\
\hline
\end{tabular}


J-1718 (5'-GGA GGA TTT GGA AAT TGA TTA GTT CC-3') and C1-N-2191 (5'-CCC GGT AAA ATT AAA ATA TAA ACT TC-3') for COI. A total volume of $50 \mu \mathrm{L}$ for each reaction contained $1 \mu \mathrm{L}$ of the genomic DNA of an individual mosquito, $1 \mathrm{X}$ PCR Buffer, $2 \mathrm{mM} \mathrm{MgCl}_{2}$, $0.2 \mathrm{mM}$ of each dNTP, $0.4 \mu \mathrm{M}$ of each primer, and 1 unit of Taq DNA polymerase. PCR cycling conditions for ND5 were as follows: a 5 minute $94^{\circ} \mathrm{C}$ denaturation step followed by 30 cycles of 45 seconds at $94^{\circ} \mathrm{C}$, 45 seconds at $46^{\circ} \mathrm{C}$ and 1 minute at $72^{\circ} \mathrm{C}$; there was a final extension step of 10 minutes at $72^{\circ} \mathrm{C}$. Thermal cycling conditions for $\mathrm{COI}$ were initial denaturation at $94^{\circ} \mathrm{C}$ for 3 minutes, 35 cycles of denaturation at $94^{\circ} \mathrm{C}$ for 30 seconds, annealing at $48^{\circ} \mathrm{C}$ for 40 seconds and extension at $72^{\circ} \mathrm{C}$ for 30 seconds, and then a final extension at $72^{\circ} \mathrm{C}$ for 10 minutes.

A total of 155 DNA samples were sent to Macrogen Inc. in Korea and sequence analysis carried out using an ABI 3730XL DNA analyzer (Applied Biosystems, Foster City, CA).

\section{Data analysis}

The DNA sequence data were aligned in Bioedit 7.0.9 [25]. The sequences were deposited in GenBank under accession numbers JQ424478-JQ424787. Sequence polymorphism and nucleotide divergence with Jukes and Cantor distance (K) statistics were estimated using DnaSP 5.0 [26]. Phylogenetic relationships for construction of a haplotype network were assessed using the statistical parsimony method implemented in TCS version 1.21 [27]. Neighbor-Joining (NJ) analysis was conducted using MEGA 4.0 [28]. Node support for NJ result was assessed using 1000 bootstrap pseudo-replicates. To find which substitution model best described the evolution of concatenated ND5 and COI sequences, Modeltest [29] was used to perform a hierarchical likelihood ratio test. The Tamura Nei model [30] was specified for concatenated ND5 and COI sequences using the Akaike information criterion. The model, TIM3 + I $+\mathrm{G}$ was used for maximum likelihood analysis using PhyML 3.0 [31]. The primer sequences were not added to the analysis due to unclear primer sequence data from some of specimens although the results were not affected.

\section{Results}

Alignments of partial sequences from the $3 /$ end of the ND5 (682 bp) and COI (524 bp) genes were analyzed from 155 individuals of An. funestus, An. funestus-like, An. parensis, An. rivulorum and An. vaneedeni identified using the Spillings et al. [11] and the Koekemoer et al. [21] methods (Table 1). All 21 An. funestus individuals from Malawi belonged to clade I while An. funestus from Mozambique showed the presence of both clades - 13
(40.6\%) for clade I and 19 (59.4\%) for clade II. The $A n$. rivulorum results were distinct from the rest of the group and this species was used as an outgroup in subsequent analyses.

\section{Haplotype network analysis}

The concatenated sequence data of ND5 and COI were analyzed using a statistical parsimony method [27] to construct an mtDNA haplotype network (Figure 1). Five main clusters emerged. The three clusters which contained An. rivulorum (Figure 1A), An. funestus-like (Figure 1B) and two An. parensis individuals excluded from the main An. parensis lineage (Figure 1C) were separated from the An. funestus subgroup lineages. The other clusters consisted of two lineages. One cluster included An. funestus clade I (Figure 1D). Mosquitoes included in clade I were within five mutational steps from the ancestral of clade I. The other cluster including An. funestus clade II (Figure 1E) was composed largely of haplotypes from An. parensis and An. vaneedeni as well as four An. funestus-like individuals (Figure 1F). There were two shared haplotypes: a) between An. funestus clade II and An. parensis; and b) between An. parensis and An. vaneedeni in Figure 1E. Anopheles funestus clade II samples were separated by multiple mutational steps from the clade I cluster. The cluster that contained the three An. vaneedeni haplotypes was located between the clade I and II clusters of An. funestus (Figure 1G).

\section{Neighbor-joining (NJ) and Maximum likelihood (ML) phylogenetic analysis}

Sequence alignments of An. funestus (GenBank access No. DQ127052 for ND5 and No. AY423059 for COI, specimens originating from Burkina Faso and Cameroon respectively) were used for the comparison in the phylogenetic trees. The concatenated sequence data of ND5 and COI (total length $1206 \mathrm{bp}$ ) for the haplotype network and phylogenetic analysis are presented due to similar results with the individual data for both genes. The concatenated sequence data were analyzed using NJ analysis (Figure 2). Again the taxa were arranged in three distinct lineages: 1) An. rivulorum, 2) An. funestuslike clade I and An. parensis clade II, and 3) An. funestus clades I and II, An. parensis clade I, An. vaneedeni clades I and II, and An. funestus-like clade II. In An. funestus from Mozambique and Malawi, the results confirmed the two subdivisions, clade I and clade II. Four $A n$. funestus-like and two An. parensis individuals that were excluded from the main species lineages (clade I) were designated An. funestus-like clade II and An. parensis clade II. Three An. vaneedeni specimens, which were separated from the main lineage (clade I), were designated An. vaneedeni clade II although they were included in the An. funestus subgroup lineage. The 

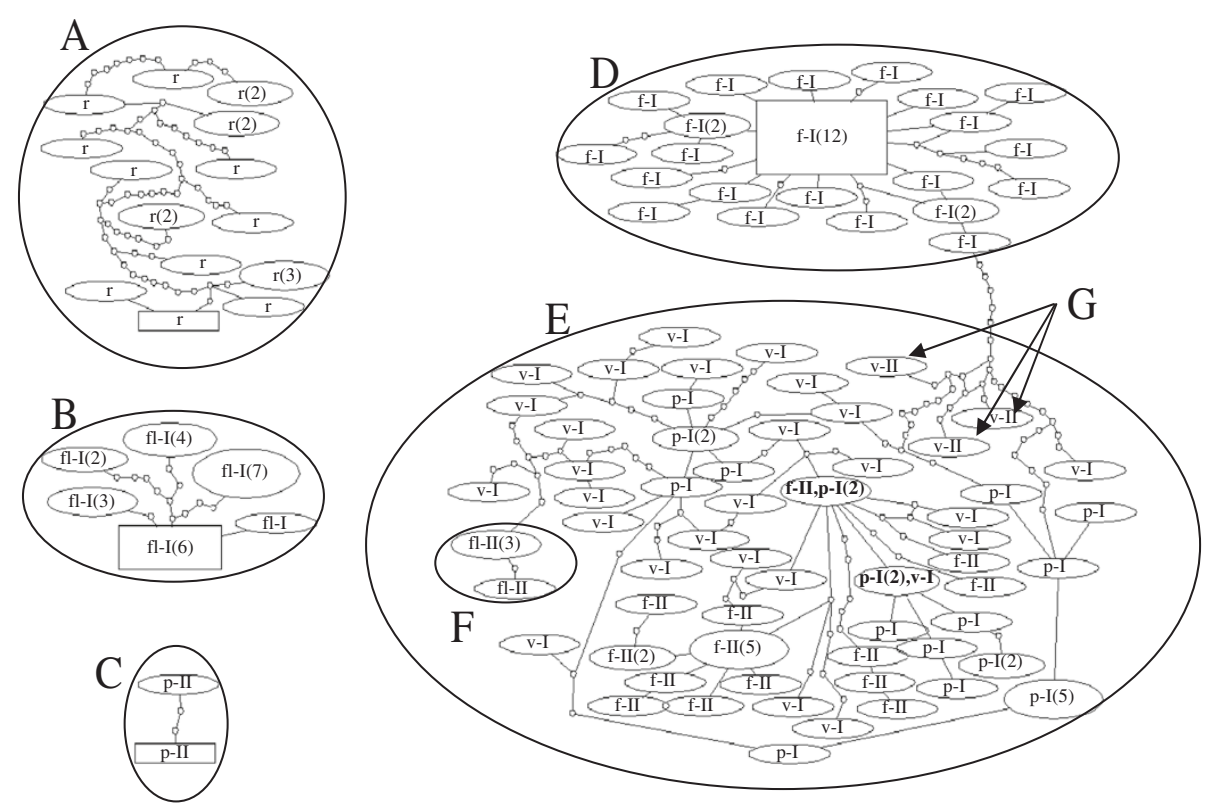

Figure 1 Haplotype networks of mtDNA ND5 and COI concatenated sequences. Haplotypes are represented as ovals, scaled to reflect frequencies. The most frequent haplotype ( $n=1$ in An. rivulorum, $n=6$ in An. funestus-like, $n=1$ in An. parensis excluded from the main An. parensis group and $n=12$ in An. funestus subgroup), inferred as ancestral, is represented by a square. Lines connecting haplotypes and small ovals indicate one mutational step. A: An. rivulorum; B: An. funestus-like; C: An. parensis excluded from the main An. parensis group in clade II; D: clade I in An. funestus; E: clade II in An. funestus, An. parensis clade I and An. vaneedeni clades I and II; F: An. funestus-like individuals excluded from the main An. funestus-like group; G: An. vaneedeni individuals excluded from the main An. vaneedeni group. f-l: An. funestus clade I; f-Il: An. funestus clade II; fl-I: An. funestus-like clade I; fl-II: An. funestus-like clade II; $\mathrm{p}$-I: An. parensis clade I; $\mathrm{p}$-II: An. parensis clade II; r: An. rivulorum; v-l: An. vaneedeni clade I; v-ll: An. vaneedeni clade II. Haplotypes marked in bold are shared between species. Figures in brackets are frequencies for each haplotype.

excluded samples were sequenced to confirm the species identification.

For the NJ tree of the An. funestus subgroup, $A n$. funestus clade I was separated from the rest (An. funestus clade II, An. parensis clade I, An. vaneedeni clades I and II, and An. funestus-like clade II) with bootstrap values of 99\%. Anopheles parensis clade II and An. funestus-like clade II were separated from An. funestus-like lineage (clade I) and An. parensis lineage (clade I) with bootstrap values of $99 \%$ and $97 \%$ respectively. Within the $A n$. funestus subgroup, An. vaneedeni clade II was separated from the main An. vaneedeni lineage (clade I) with bootstrap values of $85 \%$. The ML results are similar to the topology of the tree for the NJ results (Additional file 1).

\section{ND5 sequence analysis}

In the An. funestus group, An. rivulorum, which is the nominal member of the An. rivulorum subgroup, had the highest divergence from the other species while the nucleotide divergence with Jukes and Cantor distance (K) for An. funestus-like clade I was also high compared with the rest of the group (Table 2). Anopheles parensis clade II was separated from the An. parensis clade I (0.037) and close to An. funestus-like clade I (0.02). Table 2 shows a nucleotide divergence value of 0.023 between An. funestus clades I and II. The $\mathrm{K}$ values of An. funestus clade II against An. funestus-like clade II (0.008), An. parensis clade I (0.005), and An. vaneedeni clade I (0.006) were lower than the ones for An. funestus clade I against the same clades (Table 2). However, $A n$. vaneedeni clade II had the same values $(0.016)$ for the nucleotide divergence from both An. funestus clades. There were 9, 27 and 20 fixed different nucleotides between clades within An. funestus, An. funestus-like and An. parensis respectively (Table 3 ). Within the group, the highest polymorphic sites were found in An. vaneedeni clade I (34), and the highest number of fixed differences (27) between clades of An. funestus-like.

\section{$\mathrm{COI}$ sequence analysis}

The sequence analysis of $C O I$ between species in the $A n$. funestus group and between each two clades within $A n$. funestus, An. funestus-like, An. parensis and An. vaneedeni supported the ND5 sequence analysis although the different nucleotides between clades within An. vaneedeni were 0 for ND5 and 3 for COI (Tables 2 and 3). In the $C O I$ sequence analysis, the nucleotide divergence between $A n$. rivulorum and the other species was slightly higher than the values from the ND5 sequence analysis (Table 2). The nucleotide divergence values (K) were 


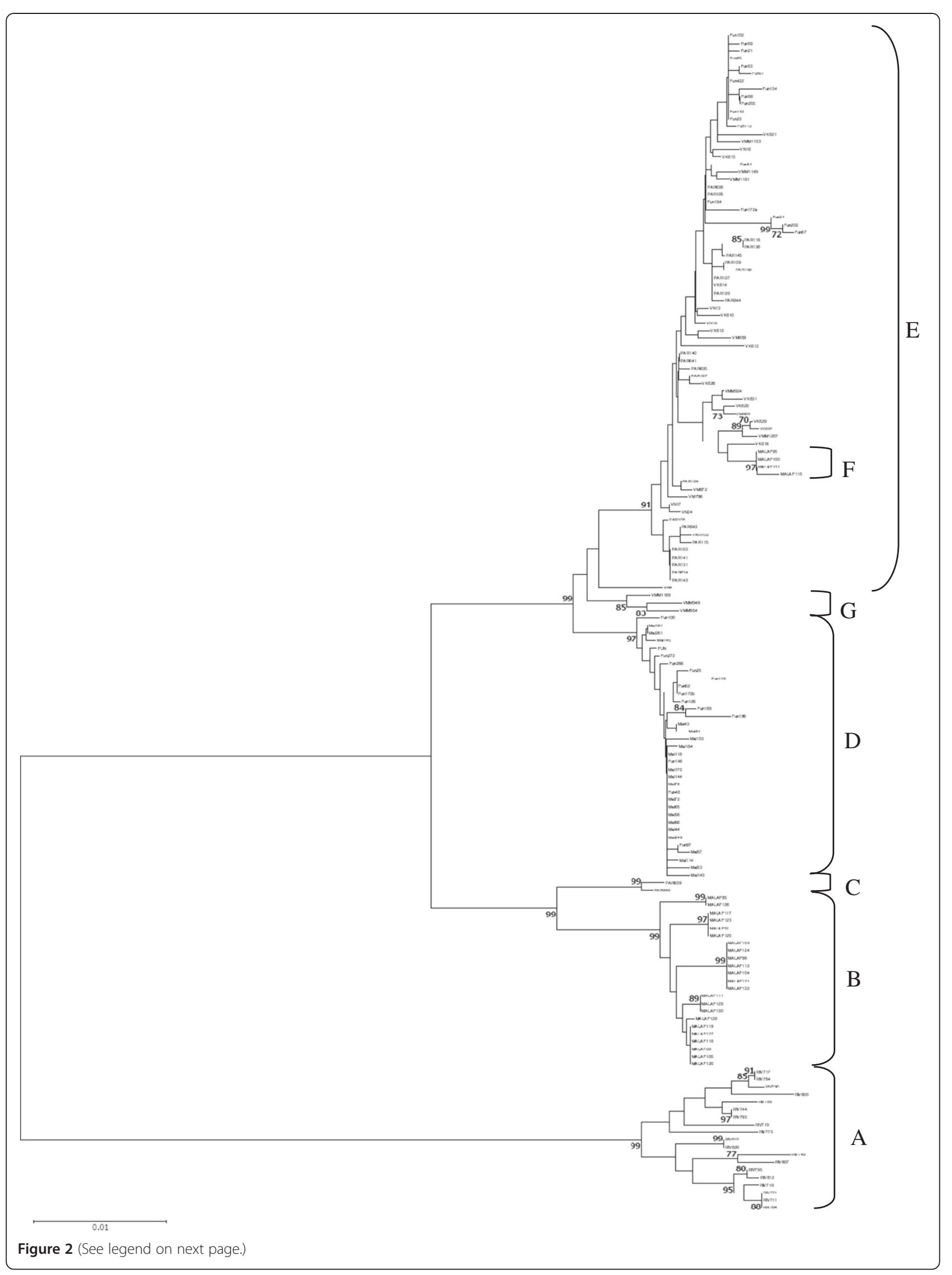


(See figure on previous page.)

Figure 2 Neighbor-joining tree. The tree inferred from the concatenated ND5 (a) and COI (b) loci with bootstrap percentages for 1,000 replicates and An. rivulorum as an outgroup. Bootstrap values under $70 \%$ are not shown. A: An. rivulorum; B: An. funestus-like clade I; C: An. parensis clade II; D: An. funestus clade I; E: An. funestus clade II, An. parensis clade I and An. vaneedeni clades I; F: An. funestus-like clade II; G: An. vaneedeni clade II.

0.019, 0.042, 0.039 and 0.017 between clades within $A n$. funestus, An. funestus-like, An. parensis and An. vaneedeni respectively. Polymorphic sites for COI between clades were lower for An. funestus (21), An. funestus-like (27), An. parensis (23) and An. vaneedeni (22) than the ones for $N D 5$ (37, 37, 29 and 36 respectively) (Table 3). Comparison of our data with the COI data of Garros et al. [32] showed that the limited samples of Garros et al. (2-3 specimens) all fell within the main lineages described here, i.e. An. funestus clade I, An. vaneedeni clade I, An. parensis clade I and An. rivulorum.

\section{Discussion}

The phylogenetic analysis of concatenated ND5 and COI genes showed the existence of three distinct lineages for the five investigated species, i.e. 1) An. rivulorum, 2) An. funestus-like clade I and An. parensis clade II, and 3) An. funestus clades I and II, An. funestus-like clade II, An. parensis clade I and An. vaneedeni clades I and II. The results presented in this study support the hypothesis that there are at least two main divisions within An. funestus which is consistent with the results of Michel et al. [15] as well as the An. funestuslike study [11].

Previous studies of the ITS2 and D3 regions of the rDNA and COI and Cytochrome Oxidase subunit II (COII) mtDNA genes, for African and Asian Anopheles groups [32,33] showed that the An. rivulorum subgroup was clearly distinguished from members of the $A n$. funestus subgroup (An. funestus, An. parensis and $A n$. vaneedeni). Although there were slight differences in distances between members of the An. funestus subgroup reported in the two studies, the results reinforce the conclusion of distinct distance between An. funestus and An. rivulorum subgroups. Garros et al. [32] suggested that An. rivulorum, which is assigned to its own subgroup within the broader An. funestus group [34] and the An. funestus subgroup might have evolved from a common ancestor based on the phylogenetic trees of ITS2, D3 and COI. It should be mentioned that An. rivulorum larvae are morphologically distinct from the members of the An. funestus subgroup [7] so it is not

Table 2 Nucleotide divergence (K) between species in the An. funestus group

\begin{tabular}{|c|c|c|c|c|c|c|c|c|c|c|c|}
\hline \multirow[t]{2}{*}{ Gene } & \multirow[t]{2}{*}{ Species } & & \multicolumn{2}{|c|}{ An. funestus } & \multicolumn{2}{|c|}{ An. funestus-like } & \multicolumn{2}{|c|}{ An. parensis } & \multirow[t]{2}{*}{ An. rivulorum } & \multicolumn{2}{|c|}{ An. vaneedeni } \\
\hline & & & 1 & II & 1 & II & 1 & II & & 1 & II \\
\hline \multirow[t]{9}{*}{ ND5 } & An. funestus & I & - & & & & & & & & \\
\hline & & $\|$ & 0.023 & - & & & & & & & \\
\hline & An. funestus-like & 1 & 0.04 & 0.044 & - & & & & & & \\
\hline & & $\|$ & 0.022 & 0.008 & 0.047 & - & & & & & \\
\hline & An. parensis & 1 & 0.021 & 0.005 & 0.044 & 0.007 & - & & & & \\
\hline & & $\|$ & 0.037 & 0.039 & 0.02 & 0.038 & 0.037 & - & & & \\
\hline & An. rivulorum & & 0.097 & 0.097 & 0.101 & 0.097 & 0.096 & 0.102 & - & & \\
\hline & An. vaneedeni & I & 0.021 & 0.006 & 0.044 & 0.008 & 0.005 & 0.038 & 0.097 & - & \\
\hline & & $\|$ & 0.016 & 0.016 & 0.039 & 0.018 & 0.013 & 0.034 & 0.097 & 0.014 & - \\
\hline \multirow[t]{9}{*}{$\mathrm{COl}$} & An. funestus & 1 & - & & & & & & & & \\
\hline & & $\|$ & 0.019 & - & & & & & & & \\
\hline & An. funestus-like & 1 & 0.032 & 0.045 & - & & & & & & \\
\hline & & $\|$ & 0.014 & 0.016 & 0.042 & - & & & & & \\
\hline & An. parensis & I & 0.013 & 0.008 & 0.04 & 0.012 & - & & & & \\
\hline & & $\|$ & 0.031 & 0.043 & 0.016 & 0.039 & 0.039 & - & & & \\
\hline & An. rivulorum & & 0.114 & 0.122 & 0.112 & 0.118 & 0.121 & 0.107 & - & & \\
\hline & An. vaneedeni & 1 & 0.014 & 0.009 & 0.04 & 0.011 & 0.007 & 0.037 & 0.12 & - & \\
\hline & & $\|$ & 0.008 & 0.022 & 0.038 & 0.017 & 0.017 & 0.033 & 0.12 & 0.017 & - \\
\hline
\end{tabular}

Samples sizes are 44 (An. funestus clade I), 19 (An. funestus clade II), 23 (An. funestus-like clade I), 4 (An. funestus-like clade II), 24 (An. parensis clade I), 2 (An. parensis clade II), 19 (An. rivulorum), 27 (An. vaneedeni clade I) and 3 (An. vaneedeni clade II). 
Table 3 Summary of fixed differences and polymorphic sites between clades in the An. funestus subgroup

\begin{tabular}{|c|c|c|c|c|c|c|c|c|c|}
\hline & & \multicolumn{2}{|c|}{ An. funestus } & \multicolumn{2}{|c|}{ An. funestus-like } & \multicolumn{2}{|c|}{ An. parensis } & \multicolumn{2}{|c|}{ An. vaneedeni } \\
\hline & & ND5 & $\mathrm{COI}$ & ND5 & $\mathrm{COI}$ & ND5 & $\mathrm{COI}$ & ND5 & $\mathrm{COI}$ \\
\hline \multirow[t]{2}{*}{ Polymorphic sites } & Within Clade I & 19 & 11 & 8 & 9 & 9 & 9 & 34 & 16 \\
\hline & Within Clade II & 17 & 7 & 2 & 0 & 2 & 1 & 5 & 5 \\
\hline Fixed differences/ Polymorphic sites & Between clades $1 / / \mid$ & $9 / 37$ & $6 / 21$ & $27 / 37$ & $18 / 27$ & $20 / 29$ & $15 / 23$ & $0 / 36$ & $3 / 22$ \\
\hline
\end{tabular}

surprising that the molecular data should reflect this separation.

The phylogenetic data for An. funestus-like in this study suggest that it is a distinct lineage from the other species in the group. These results support Spillings et al. [11] showing this to be a new member of the $A n$. funestus group. However, the phylogenetic trees from the concatenated ND5 and COI both showed two clades for $A n$. funestus-like. Four An. funestus-like specimens were separated into their own lineage with the rest falling into the $A n$. funestus subgroup lineage. Furthermore, two $A n$. parensis specimens were separated from the main $A n$. funestus subgroup lineage and grouped with the $A n$. funestus-like lineage. Further molecular investigations are needed to test the hypothesis of new species in the group and determine the relationship between $A n$. funestus-like and the two An. parensis individuals.

In the study by Michel et al. [15], samples from East Africa had significantly lower average heterozygosity (0.455) and allelic richness (3.9) across all microsatellite loci, and lower mean mtDNA haplotype diversity (0.773) compared with the rest of Africa (0.606, 6.0 and 0.924 respectively). Other studies [16,35] have also reported two different subdivisions within An. funestus from the analysis of ND5. However, these proposed subdivisions were not correlated with the clades of Michel et al. [15]. Analysis of the mtDNA cytochrome $b$ gene and the ITS2 region in the rDNA [36] did not find any subdivisions within An. funestus. Previous studies using the RFLP method [17,18] that included samples of An. funestus from 16 African countries, found evidence for five genetic subdivisions on the ITS2 and D3 regions in the rDNA but again these were not correlated with the Michel et al. [15] clades.

In the phylogenetic trees in this study, the results did not show clear phylogeny between An. funestus clade II, $A n$. parensis clade I and An. vaneedeni in the An. funestus subgroup even though these three were separated clearly from $A n$. funestus clade I. This undistinguishing relationship between the three species may reflect a selective sweep or non-discrimination due to recent divergence that is known to occur in the mtDNA [37].

Surprisingly, An. parensis from South Africa shared two of the haplotypes, one with An. funestus from Mozambique and one with $A n$. vaneedeni from South Africa. Donnelly et al. [38] reported that shared haplotypes between species in the An. gambiae complex might reflect non-contemporary processes such as incomplete lineage sorting between species or historical introgression events. So, although no natural hybridization between An. funestus clade II, An. parensis and An. vaneedeni has been reported, mitochondrial introgression may have happened through a recent event. However, Green and Hunt [39] reported that cross-mating experiments between $A n$. vaneedeni and $A n$. funestus resulted in sterile male hybrids and asynapsis of the giant polytene chromosomes, two phenomena that occur regularly in crosses between species of Anopheles [11,19,40]. A more likely explanation, therefore, is that these shared haplotypes are ancestral.

Although there were apparent subdivisions in the species studied here, these divergences may only be limited to mtDNA. The nature of mtDNA, i.e. haploid, maternal heritage and non-recombination, could retain both distinct mtDNA clades in admixed populations, while their nuclear genome would become homogenized. Michel et al. [15] reported that there was no corresponding nuclear divergence in spite of deep mtDNA divergence between clades I and II within An. funestus. They suggested that the subdivisions may result from historical introgression either among previously isolated and divergent populations or with a related species [15]. Additional research from other genomic regions is required to determine what these results mean in terms of specific status and relevance in epidemiology and to investigate their roles in malaria transmission in order to better understand the ecological aspects of this important vector group.

\section{Conclusions}

Our findings indicate that five species in the An. funestus group comprises three lineages: A) An. rivulorum, B) An. funestus-like clade I and An. parensis clade II, and C) An. funestus clades I and II, An. funestus-like clade II, An. parensis clade I and An. vaneedeni clades I and II. This is the first step in the phylogenetic reconstruction of relationships between $A n$. funestus-like and the other four common species of the An. funestus group, as well as between clades I and II of An. funestus. The study supports the conclusion that An. funestus-like is a new member of the An. funestus subgroup [11]. Intriguingly, the phylogenetic trees of the concatenated ND5 and COI 
sequences showed that two subdivisions each for $A n$. funestus-like, An. parensis and An. vaneedeni exist. Further investigations will be carried out to determine the specific status of the clades. In addition, further studies should also give insight into the roles played by the various species in malaria transmission.

\section{Additional file}

Additional file 1: Maximum-likelihood tree. The tree inferred from the concatenated ND5 (a) and COI (b) loci with bootstrap percentages for 1,000 replicates and An. rivulorum as an outgroup. Bootstrap values under 70\% are not shown. A: An. rivulorum; B: An. funestus-like clade I; C: An. parensis clade II; D: An. funestus clade I; E: An. funestus clade II, An. parensis clade I and An. vaneedeni clades I; F: An. funestus-like clade II; G: An. vaneedeni clade II.

\section{Competing interests}

The authors declare no conflict of interest.

\section{Authors' contributions}

KSC carried out the experiments and the analysis of the data and drafted the manuscript. MC and LLK designed the study, assisted with analysis of the data and helped draft the manuscript. All authors read and approved the final version of the manuscript.

\section{Acknowledgements}

This work was supported by the Department of Science and Technology, National Research Foundation Research Chair Initiative grant to MC and the Hillel Friedland Fellowship to KSC. We thank the following for assistance with field collections or supply of specimens: A. Mabuza (Department of Health, Mpumalanga Province, South Africa); K. Hargreaves (Department of Health, KwaZulu/Natal Province, South Africa); T. Ledwaba (Department of Health, Limpopo Province, South Africa) and Prof R.H. Hunt (University of the Witwatersrand). Dr MJ Donnelly, Dr NA Dyer and two anonymous reviewers are thanked for helpful criticisms of the manuscript.

Received: 26 July 2012 Accepted: 28 November 2012

Published: 6 December 2012

\section{References}

1. World Health Organization: The world malaria report 2011. Geneva: World Health Organization; 2011.

2. Harling $R$, Crook P, Lewthwaite P, Evans M, Schmid ML, Beeching NJ: Burden and cost of imported infections admitted to infectious diseases units in England and Wales in 1998 and 1999. J Infect 2004, 48:139-144.

3. Greenwood B, Mutabingwa T: Malaria in 2002. Nature 2002, 415:670-672.

4. Hargreaves K, Koekemoer LL, Brooke BD, Hunt RH, Mthembu J, Coetzee M: Anopheles funestus resistant to pyrethroid insecticides in South Africa. Med Vet Entomol 2000, 14:181-189.

5. Hemingway J, Ranson $\mathrm{H}$ : Insecticide resistance in insect vectors of human disease. Annu Rev Entomol 2000, 45:371-391.

6. Ranson H, Abdallah H, Badolo A, Guelbeogo WM, Kerah-Hinzoumbe C, Yangalbe-Kalnone E, Sagnon N'f, Simard F, Coetzee M: Insecticide resistance in Anopheles gambiae: data from the first year of a multicountry study highlight the extent of the problem. Malar J 2009, 8:299.

7. Gillies MT, de Meillon B: The Anophelinae of Africa South of the Sahara, Volume 54. Johannesburg: Publications of the South African Institute for Medical Research; 1968.

8. Harbach RE: The classification of genus Anopheles (Diptera: Culicidae): a working hypothesis of phylogenetic relationships. Bull Entomol Res 2004, 94:537-553.

9. Gillies MT, Coetzee M: A Supplement to the Anophelinae of Africa South of the Sahara (Afrotropical Region), Volume 55. Johannesburg: Publications of the South African Institute for Medical Research; 1987.

10. Couhet A, Simard F, Toto J-C, Kengne P, Coetzee M, Fontenille D: Species identification within the Anopheles funestus group of malaria vectors in
Cameroon and evidence for a new species. AmJTrop Med Hyg 2003, 69:200-205.

11. Spillings BL, Brooke BD, Koekemoer LL, Chiphwanya J, Coetzee M, Hunt RH: A new species concealed by Anopheles funestus Giles, a major malaria vector in Africa. AmJTrop Med Hyg 2009, 81:510-515

12. Wilkes TJ, Matola YG, Charlwood JD: Anopheles rivulorum, a vector of human malaria in Africa. Med Vet Entomol 1996, 10:108-110.

13. De Meillon B, Van Eeden GJ, Coetzee L, Coetzee M, Meiswinkel R, Du Toit LN, Hansford CF: Observations on a species of the Anopheles funestus subgroup, a suspected exophilic vector of malaria parasites in northeastern Transvaal. South Africa. Mosq News 1977, 37:657-661.

14. Hackett BJ, Gimnig J, Guelbeogo W, Costantini C, Koekemoer LL, Coetzee M, Collins FH, Besansky NJ: Ribosomal DNA internal transcribed spacer (ITS2) sequences differentiate Anopheles funestus and An. rivulorum, and uncover a cryptic taxon. Insect Mol Biol 2000, 9:369-374.

15. Michel AP, Ingrasci MJ, Schemerhorn BJ, Kern M, Le Goff G, Coetzee M, Elissa N, Fontenille D, Vulule J, Lehmann T, Sagnon N, Costantini C, Besansky $\mathrm{NJ}$ : Rangewide population genetic structure of the African malaria vector Anopheles funestus. Mol Ecol 2005, 14:4235-4248.

16. Michel AP, Guelbeogo WM, Grushko O, Schemerhorn BJ, Kern M, Willard MB, Sagnon N, Costantini C, Besansky NJ: Molecular differentiation between chromosomally defined incipient species of Anopheles funestus. Insect Mol Biol 2005, 14:375-387.

17. Garros C, Koekemoer LL, Kamau L, Awolola TS, Van Bortel W, Coetzee M, Coosemans M, Manguin S: Restriction fragment length polymorphism method for the identification of major African and Asian malaria vectors within the Anopheles funestus and An. minimus group. AmJTrop Med Hyg 2004, 70:260-265

18. Koekemoer LL, Kamau L, Garros C, Manguin S, Hunt RH, Coetzee M: Impact of the Rift Valley on restriction fragment length polymorphism typing of the major African malaria vector Anopheles funestus (Diptera: Culicidae). J Med Entomol 2006, 43:1178-1184.

19. Coetzee M, Koekemoer LL: Molecular systematics and insecticide resistance in the major African malaria vector. Anopheles funestus. Annu Rev Entomol 2013, 58:393-412.

20. Coetzee M, Fontenille D: Advances in the study of Anopheles funestus, a major vector of malaria in Africa. Insect Biochem Mol Biol 2004, 34:599-605.

21. Koekemoer LL, Kamau L, Hunt RH, Coetzee M: A cocktail polymerase chain reaction assay to identify members of the Anopheles funestus (Diptera: Culicidae) group. AmJTrop Med Hyg 2002, 66:804-811.

22. Choi KS, Coetzee M, Koekemoer LL: Simultaneous identification of the Anopheles funestus group and Anopheles longipalpis type C by PCR-RFLP. Malar J 2010, 9:316.

23. Ballinger-Crabtree ME, Black WC, Miller BR: Use of genetic polymorphisms detected by the Random-Amplified Polymorphic DNA Polymerase Chain Reaction (RAPD-PCR) for differentiation and identification of Aedes aegypti subspecies and populations. AmJTrop Med Hyg 1992, 47:893-901.

24. Simon C, Frati F, Beckenbach A, Crespi B, Lui H, Flook P: Evolution, weighting and phylogenetic unity of mitochondrial gene sequences and a compilation of conserved polymerase chain reaction primers. Ann Entomol Soc Am 1994, 87:651-701.

25. Hall TA: Bioedit: a user-friendly biological sequence alignment editor and analysis program for Windows 95/98/NT. Nucleic Acids Symp 1999, Series 41:95-98.

26. Rozas J, Sanchez-Delbarrio JC, Messeguer X, Rozas R: DnaSP, DNA polymorphism analyses by the coalescent and other methods. Bioinformatics 2003, 19:2496-2497.

27. Clement M, Posada D, Crandall KA: TCS: a computer program to estimate gene genealogies. Mol Ecol 2000, 9:1657-1659.

28. Tamura K, Dudley J, Nei M, Kumar S: MEGA4: Molecular Evolutionary Genetics Analysis (MEGA) software version 4.0. Mol Biol Evol 2007, 24:1596-1599.

29. Posada D, Crandall KA: Modeltest: testing the model of DNA substitution. Bioinformatics 1998, 14:817-818.

30. Tamura K, Nei M: Estimation of the number of nucleotide substitutions in the control region of mitochondrial DNA in humans and chimpanzees. Mol Biol Evol 1993, 10:512-526.

31. Guindon S, Dufayard JF, Lefort V, Anisimova M, Hordijk W, Gascuel O: New algorithms and methods to estimate maximum-likelihood phylogenies: Assessing the performance of PhyML 3.0. Syst Biol 2010, 59:307-321.

32. Garros C, Harbach RE, Manguin S: Systematics and biogeographical implications of the phylogenetic relationships between members of the 
Funestus and Minimus groups of Anopheles (Diptera: Culicidae). J Med Entomol 2005, 42:7-18.

33. Garros C, Harbach RE, Manguin S: Morphological assessment and molecular phylogenetics of the Funestus and Minimus groups of Anopheles (Cellia). J Med Entomol 2005, 42:522-536.

34. Harbach RE: Review of the internal classification of the genus Anopheles (Diptera: Culicidae): the foundation for comparative systematics and phylogenetic research. Bull Entomol Res 1994, 84:331-342.

35. Michel AP, Grushko O, Guelbeogo WM, Lobo NF, Sagnon N: Divergence with gene flow in Anopheles funestus from the Sudan savanna of Burkina Faso. West Africa. Genetics 2006, 173:1389-1395.

36. Mukabayire O, Boccolini D, Lochouarn L, Fontenille D, Besansky NJ: Mitochondrial and ribosomal internal transcribed spacer (ITS2) diversity of the African malaria vector Anopheles funestus. Mol Ecol 1999, 8:289-297.

37. Bazin $\mathrm{E}$, Glemin $\mathrm{S}$, Galtier N: Population size does not influence mitochondrial genetic diversity in animals. Science 2006, 312:570-572

38. Donnelly MJ, Pinto J, Girod R, Besansky NJ, Lehmann T: Revisiting the role of introgression vs shared ancestral polymorphisms as key processes shaping genetic diversity in the recently separated sibling species of the Anopheles gambiae complex. Heredity 2004, 92:61-68.

39. Green CA, Hunt RH: Interpretations of variation in ovarian polytene chromosomes of Anopheles funestus Giles, A. parensis Gillies, and A. aruni ? Genetica 1980, 51:187-195.

40. Davidson G, Paterson HE, Coluzzi M, Mason GF, Micks DW: The Anopheles gambiae complex. In Genetics of Insect Vectors of Disease. Edited by Wright JW, Pal R. Amsterdam: Elsevier Publishers; 1967:211-250.

doi:10.1186/1756-3305-5-283

Cite this article as: Choi et al: Population genetic structure of the major malaria vector Anopheles funestus s.s. and allied species in southern Africa. Parasites \& Vectors 2012 5:283.

\section{Submit your next manuscript to BioMed Central and take full advantage of:}

- Convenient online submission

- Thorough peer review

- No space constraints or color figure charges

- Immediate publication on acceptance

- Inclusion in PubMed, CAS, Scopus and Google Scholar

- Research which is freely available for redistribution 